\title{
Forensic Implications of Foot Arch Index Comparison between Dynamic Bare Footprints and Shoe Insole Foot Impressions Novelty Statement
}

The arch of a footprint provides information about the maker's anatomy, biomechanics, weight, sex, possible trauma of the lower extremity, and anthropological information. Footwear and footprints have important evidentiary value in forensic settings. While research has been done on arch indices, no research could be found that has focused exclusively on examining the changes, if any, on the impression of foot's arch on the insole of an athletic shoe, particularly in the forensic context. In this study the authors aim to compare arch height indices between bare footprints and athletic shoe insole foot impressions to determine if an association for estimation of one from the other can be found. 
Forensic Implications of Foot Arch Index Comparison between Dynamic Bare Footprints and Shoe Insole Foot Impressions Highlights

- Footprints and insole impressions were compared for significant relationships

- The Chippaux-Smirak and Staheli arch indices were used to assess arch impressions

- No significant relationships were found between barefoot and insole arch indices

- Barefoot arch height cannot be predicted from an insole arch height or vice versa 


\section{Forensic Implications of Foot Arch Index Comparison between Dynamic Bare Footprints and Shoe Insole Foot Impressions}

\section{CONFLICT OF INTEREST STATEMENT}

The authors whose names are listed immediately below certify that they have NO affiliations with or involvement in any organization or entity with any financial interest (such as honoraria; educational grants; participation in speakers' bureaus; membership, employment, consultancies, stock ownership, or other equity interest; and expert testimony or patent-licensing arrangements), or non-financial interest (such as personal or professional relationships, affiliations, knowledge or beliefs) in the subject matter or materials discussed in this manuscript.

$$
\text { Michael Nirenberg, DPM }
$$

Clinical and Forensic Podiatrist, Friendly Foot Care, PC, Crown Point, Indiana, USA

$$
\text { Elizabeth Ansert, DPM, MA }
$$

Podiatric Resident, St. Vincent Hospital, Worcester, Massachusetts, USA

Jackie Campbell, PhD MSc BSc CStat CPhys FRCOT FRSB

Professor of Neurophysiology, University of Northampton, Northampton, UK

Michael Curran, Ph.D, M.Phil, MBA, BSc(Hons)

Associate Professor Podiatry, University of Northampton, Northampton, UK

\section{Correspondence address:}

Friendly Foot Care, PC

50 West 94th Place

Crown Point, IN 46307

219-663-2273

Info@friendlyfootcare.com 


\section{Original Research Paper}

Forensic Implications of Foot Arch Index Comparison between Dynamic Bare Footprints and Shoe Insole Foot Impressions

\section{Introduction}

Foot-related evidence may be found at crime scenes in the form of footprints, partial footprints or a foot impression on the insole of footwear. This evidence can assist in linking or unlinking a suspect to the crime [1, 2]. The forensic value of this foot-related evidence is the result of the individuality of footprints [1-11]. Though papillary ridges that may be seen in a footprint are suggested to be as individual as those in fingerprints, the authors could not find any legal case or scientific study referencing them on footwear insoles [4]. In the absence of such ridges, a footprint is still distinctive due to a variety of factors that impact the formation of a footprint, such as morphology, anatomy, size, biomechanics, possible pathology, and other features of the foot [1-11].

These factors may have a role in the formation of a foot impression on a shoe's insole, though the shoe itself will be a factor. Research by Hammer et al discussed the possibility of the shoe constricting the foot, citing the example of the toe positions being altered potentially [12]. Nirenberg et al studied linear measurement comparison between bare footprints and foot impressions on athletic insoles and found linear measurements correlated with each other [13]. The study suggested that insoles are comparable to footprints when using the Reel two-dimensional linear analysis. However, it is noted that significant differences were observed for the heel to the tip of the first digit length, heel to left fifth digit length and calcaneal width. The authors could not find 
any other scientific studies showing a width discrepancy between bare footprints and foot impressions on footwear insoles, though research in this area is scant [13].

While a footprint occurs in an instant, a foot impression on a shoe insole occurs over time as the foot and shoe interact. This is a combination of the individual using his foot to walk (i.e. dynamic foot impressions) and stand (i.e. static foot impressions) [1]. The foot impression on a shoe insole may also form in part due to a specific activity that the person engages in where they are neither standing still nor walking, such as someone who needs to regularly work a machine with their foot.

The arch of a footprint provides information about the maker's anatomy, biomechanics, weight, sex, possible trauma of the lower extremity, and anthropologic information [15-20]. The discriminatory value of arch indices has also been used in larger scale studies, such as to predict musculoskeletal health [21], injury in athletes [18], and flatfoot diagnosis [22, 23].

Queen et al [15] used footprint arch indices to estimate the medial column height of the foot, which significantly contributes to lower extremity biomechanics. The study also examined the reliability of several indices and found that the Staheli and ChippauxSmirak were among the most reliable techniques. Wrobel et al found Chippaux-Smirak and Staheli arch indices to be moderately reliable [24]. Anthropological differences have been inferred from arch index studies. For example, Echarri and Forriol [25] studied arch index differences between rural and urban raised children. They found that boys were more likely to have flat feet and the use of footwear did not affect the morphology of the foot. 
Though the methodology originally described for these arch indices considered standing footprints (i.e. static) more recent research has shown the reliability for the Staheli Arch Index and the Chippaux-Smirak Index to be reliable for footprints created while walking (i.e. dynamic) [26]. Dynamic footprints generally have larger linear dimensions. $[3,26,27,28]$

Ideally, foot impressions on a shoe or boot insole should be evaluated by comparison with a foot impression from a similar shoe or boot from the same owner [1, 13]. This methodology was utilized by podiatrist Norman Gunn in the 1980s. He compared a foot impression on the insole of a shoe recovered from a crime scene to that of a foot impression on the suspect's shoe insole [14]. The comparison process is referred to as a "like to like" analysis [1].

A "like to like" comparison is considered the most precise of comparison, but evaluation of the suspect's footprint to the shoe insole is suggested as an additional component of the forensic analysis of the suspect to an unknown shoe [1]. There may also be situations where the suspect's shoe may not be available or its style may differ from the shoe found at the crime scene. Both Nirenberg et al and Hammer et al.'s research recommended using this "like to like" methodology when possible $[12,13]$.

While research has been done on arch indices, no research was found that focused on examining the arch of dynamic foot impressions on insoles of athletic shoes in the forensic context. Yet, footwear has been found at crime scenes. In one case the murderer's shoe fell off during a scuffle with the victim and the linking of the shoe to the suspect was presented as evidence by a forensic podiatrist at trial [29, 30]. During a sexual assault and murder case, the victim was killed while on a beach. The crime 
scene did not have any footprints; however, the perpetrator left his shoes behind. Footprint impressions recovered from both shoe insoles helped link the shoes to a suspect. [31]

In this study, the authors aim to compare arch height indices between bare dynamic footprints and athletic shoe insole foot impressions to determine if an association for estimation of one from the other can be found.

\section{Materials and Methods}

\subsection{Design}

The present study is a quantitative index comparison of two-dimensional footprints taken on a solid surface with foot impressions on the corresponding shoe insole. Participants gave written consent to participate. The study followed the ethical standards described in the 1964 Helsinki Declaration and its amendments. Some participants took part in previous forensic podiatry related research [13, 32, 33].

\subsection{Materials}

Dynamic footprints were collected using the Identicator® Inkless Shoe Print Model LE25P system (Fig. 1). Dynamic footprints, as opposed to static footprints, were collected because they more closely approximate the dynamic component involved in the formation of the foot impression on a shoe insole. Participants stepped onto the system's coater first and then onto the system's impression paper. The chemical on the coating created a black exemplar of the participant's footprint impression on the paper. The insoles that were collected from the participants' shoes had to have a foot impression visible to the naked eye. The importance of this visibility of the foot impression was for enhancement 
within the GIMP program for accurate and reliable measurement. All participants stated they had worn the shoes for at least 6 months.

The coater was cleaned with antibacterial wipes before and after each set of participant footprints were collected. Each participant cleaned their foot before the trial and was offered the option of cleaning their feet after the trial. The walking area was swept to remove any debris and cleaned using antibacterial wipes or a Swiffer Wet Jet before and after each participant. These steps were taken to ensure that any microbes or contaminants were not transmitted between participants.

\subsection{Methods}

A mid-gait protocol was employed for participants to utilize their most natural walking gait [34, 35]. Participants were allotted a length of seven meters of hard, flat surface to walk. Starting with their right foot, each participant took ten steps from the designated starting point, as demarked by a piece of tape. Participants returned to the starting point with a natural walking stride. All participants were able to take full, natural strides. This was repeated three times. The position of the right foot for the participant's third and fifth step were marked by the researchers using paper markers. The inkless coater from the Identicator ${ }^{\circledR}$ was positioned at the marked locations of the participant's third step. The impression sheets were placed at the fifth step [36]. The impression sheets were secured with tape on the outermost corners to allow for the largest possible surface area to be exposed. This procedure was repeated until a footprint entailing the entire foot was created. This method was used to collect a footprint of the left but used the fourth and sixth steps of the gait cycle. 
Once the participant's footprints were obtained, the insoles from the participant's shoes were removed. A PDF image of each footprint on the impression paper and each insole was created by using the scanning function of the Konica Minolta Bizhub 4052 series printer or an HP Envy 4520 series printer.

The scanning of the shoe insoles did not involve closing the lid of the scanner over the insoles to the point of causing compression of the insole or placing significant weight on the insole but instead only gently resting the lid on each insole in order to reduce the amount of light entering while the image was scanned. This procedure allowed recording of the insoles while not distorting the foot impressions on them.

The footprint and corresponding insole were scanned on the same printer. The PDF images were labeled and saved onto the Apple MacBook Pro. This procedure was repeated until 122 bare footprints and insoles were collected (56 right and 56 left footprints).

The GIMP (GNU Image Manipulation Program) on the same Apple MacBook Pro allowed for the distances between anatomical landmarks for each bare footprint and shoe insole impression to be measured in centimeters. GIMP is open source software that has been shown to be reliable for analysis of footprints [33, 37]. While no significant differences between GIMP, Photoshop, or manual measurement techniques have been found, two-dimensional measurement was originally performed on GIMP software due to its wide availability and user-friendly qualities $[33,37]$. Though each insole had a foot impression that was visible to the naked eye, the visibility of foot impression insole scans were improved with GIMP's contrast and/or brightness adjustments. These adjustments did not alter the size or dimensions of the foot impression on the insole. 
2.4 Analysis of the Footprints and Insole Impressions

To measure each bare footprint and insole foot impression, the widest parts of the forefoot and heel were measured in centimeters using GIMP software on an Apple Mac Pro laptop. Anecdotally, the constraint of the shoe on the foot could theoretically limit the width of the footprint impression, the only research addressing this issue that could be found by the authors was that done by Nirenberg et al, which found narrowing of the width of the heel only [13]. The narrowest aspect of the midfoot was measured in the same way. The measurements were recorded in a Microsoft excel spreadsheet on the same computer. The Staheli arch index and Chippaux-Smirak index were used to assess the arch of the bare footprints based on their reliability and user-friendly aspects. While the Clarke's Arch Index was considered, difficulty assessing this index on GIMP software raised concern about its accuracy, causing the authors to exclude its use. The Staheli arch index was found to have an interrater reliability of 0.963 , and ChippauxSmirak index was determined to have a 0.961 interrater reliability by Queen et al and these measurements have also been used in other research areas to quantify arch height and characteristics [15].

Once all footprints and insole foot impressions were measured, the Staheli and Chippaux-Smirak arch indices were calculated by midfoot width divided by the heel and forefoot widths, respectively. The heel width and forefoot width were measured from the most medial to the most lateral aspects of the print. Figures 1 and 2 show the labeled bare footprint and shoe insole respectively.

2.5 Statistical Analysis 
Linear regression analysis was used to investigate the relationship between arch height indices calculated from measurements taken from barefoot and insole prints. Both Staheli (midfoot width/heel width) and Chippaux-Smirak (midfoot width/forefoot width) arch height indices were investigated. Measurements were taken from both left and right feet of the participants. For this analysis, it was assumed that these were independent measures as, though there is less variance in left-right measures within participants than between participant, the barefoot and insole measures were only being compared within the same foot, with no left-right comparisons. Data for prints with incomplete arches were not included.

\section{Results}

\subsection{Sample characteristics}

56 participants were invited to participate in the study. Participants were volunteers from podiatric medical schools or residents in a three-year podiatric surgical program located in the United States. A total of 38 males and 18 females participated in the study with an average age of 28.1 years old and age range of 22 to 61 years old. 28 participants were Caucasian, 15 Asian, 9 Hispanic, 2 African American, and 2 who did not identify their race. Participants with an obvious limb or foot abnormality, deformity, or pathology were excluded from the study. 49 participants had complete arch prints for at least one foot (35 for both feet, 14 for one foot), giving a total of 84 feet from which forefoot, midfoot and heel width measurements were taken, and arch height indices calculated. Of these 49 participants, $13(32 \%)$ were female and $27(68 \%)$ male. 1 had missing demographic data. Their ages ranged from 23 to 61 years but were skewed 
towards the younger ages with a median age of 26 years (IQR 25-28 years). A sample size of 84 achieves $80 \%$ power to detect a difference of $0.3\left(R^{2}=0.09\right)$ between the null hypothesis correlation of 0.0 and an alternative hypothesis correlation of 0.3 using a two-sided hypothesis test with a significance level of 0.05 .

\subsection{Regression analysis}

Simple linear models were constructed for each of the two arch height indices, with the barefoot arch height index as the outcome variable and the insole arch height index as predictor. Assumptions of the linear model were tested and confirmed. Statistical significance was indicated if $p<0.05$.

\subsubsection{Staheli Arch Index:}

The correlation coefficient between the Staheli index for insole and barefoot measurements was very low and not statistically significantly different from 0 ( $r=-0.01$, $p=0.92$ ). Less than $0.1 \%$ of the variation seen in the barefoot Staheli index can be accounted for by the equivalent insole value. The regression analysis indicated that the insole Staheli index was not a statistically significant predictor of the barefoot Staheli index $(F(1,82)=0.01, p=0.92)$. Figure 3 illustrates the relationship between the insole and barefoot-derived Staheli indices. It can be seen that the best fit line is almost horizontal, at approximately a value of 0.58 . The dotted lines indicate the $95 \%$ confidence interval for prediction of the barefoot index from the insole value and it can be seen that these are extremely wide (approximately \pm 0.31 ) and include almost all data points, with the exception of 5 outliers.

\subsubsection{Chippaux-Smirak Index:}


The analysis above was repeated using the Chippaux-Smirak arch indices calculated from barefoot and insole prints as outcome and predictor variables respectively. The correlation coefficient was again extremely small and not statistically significantly different from $0(r=0.06, p=0.56)$, indicating that the insole measure could only explain $0.4 \%$ of the variation in the barefoot measures. The regression model was not a statistically significant predictor of the barefoot arch index $(F(1,82)=0.34, p=0.56)$. Figure 4 illustrates the relationship between the insole and barefoot-derived ChippauxSmirak indices. It can be seen that the best fit line is almost horizontal, with only a very small positive gradient at approximately a value of 0.3 . The dotted lines indicate the $95 \%$ confidence interval for prediction of the barefoot index from the insole value and it can be seen that these are very wide (approximately \pm 0.16 ) and include almost all data points, with the exception of 4 outliers.

\subsection{Results}

Overall, the regression analyses show that the arch height of a barefoot print as measured by either the Staheli or Chippaux-Smirak arch height indices are statistically uncorrelated and the barefoot arch height index cannot be predicted from the equivalent arch height index from an insole foot impression.

\section{Discussion}

The aim of the current study was to examine the similarities and/or differences seen between dynamic bare footprint arch indices and the arch indices found on athletic shoe insole foot impressions. Overall, no statistically significant relationship or correlation was found. 
An explanation for a lack of correlation could be a result of athletic shoes design and function. To reduce repetitive loading force, athletic shoes often are constructed with thick, durable material that may not allow for impact forces with the ground to be distributed across the foot in the same way that occurs when the foot is bare [38]. It can be deduced that the construction of each participant's particular athletic shoe combined with the specific anatomy and biomechanics of the subject's foot act together to affect the arch impression formed on the insole, which might account for the lack of correlation. Also, the particular material of a given athletic shoe may conform to a varying degree to a person's foot and alter the arch impression made on that shoe's insole [39]. Most shoes, especially athletic shoes, change the natural gait of the participant by trying to correct for abnormal or excessive movements, which may influence the arch impression [40]. Last, it is possible that one or more of the parameters used in determining the arch index are not accurately reflected in the foot impression on the shoe insole. For example, the rigid heel cup in an athletic shoe may not allow for the expansion of the heel's fat pad, therefore distorting the measurement of the Staheli arch index.

While the authors could not find any research comparing arch index of bare footprints and footprint impressions on shoe insoles in the forensic context, bare footprints and foot impressions on shoe insoles have been examined. As noted, Nirenberg et al found no statistical differences for all linear measurements from the Reel two-dimensional methodology, except for the calcaneal width and length from heel to the $5^{\text {th }}$ toe [13]. This was supported by Hammer et al who demonstrated that shoe insole impressions were similar to bare footprints in a "like versus like" comparison 
when made by the same person [12]. While both studies compare bare footprints to foot impressions on shoe insoles, neither specifically studied the arch of bare footprints or insole foot impressions.

Based on Nirenberg et al's finding of a significant heel width difference between foot impressions on shoe insoles and bare footprints, more caution should be applied to comparisons involving the Staheli arch index, which may be less accurate than the Chippaux-Smirak arch index.

Though arch studies of footprints have been shown to provide valuable information about the donor's anatomy, biomechanics, weight, sex, and possible trauma of the lower extremity [15-20], no studies could be found that were done in a forensic context. As the present study may be the first to consider arch index measurements in the forensic context, caution should be used when applying these findings to criminal matters. The authors suggest that the forensic expert utilizing the arch index when comparing footprints to foot impressions on shoe insoles should consider incorporating other methods of footprint comparison, such as linear measurements or the overlay method [1]. Though not a finding of this study, the authors speculate that differences in the arch index between a bare footprint and a foot impression on a shoe insole may occur in cases of more pronounced arch deformity, such as in a person with a very low arched foot or a very high arched foot.

Limitations exist within the current study. The arch indices used in this study provide only a generalization of arch height, reducing discrimination. All participants were asked to bring the insoles of their everyday athletic shoes for the study. As previously mentioned, different materials are used by different companies, which may 
affect the arch impressions. In future studies, consideration of the material, brand of athletic shoe, or different shoe types might be included in the protocol or examined.

Another limitation is that participants wore socks with their shoes. While socked footprints have been shown to have similar linear measurements as bare footprint impressions [32], the authors could not find any studies evaluating the relationship between the arch of a given individual's bare and sock-clad footprint. Also, the wearing of a sock may affect the arch impression on a shoe insole, and this may be an avenue for further study.

Last, a larger sample size should be considered, as well as studying populations of participant with more pronounced arch deformity, such as a flat foot or high arched foot, as this may yield significant differences.

\section{Conclusion}

The findings from this study showed that the arch height of a bare footprint and a footprint impression on the insole of an athletic shoe as measured by either the Staheli or Chippaux-Smirak arch height indices are statistically uncorrelated. The findings of this study may assist forensic podiatrists and other forensic experts in analyzing shoe insole evidence.

In the forensic context, footprint analysts considering arch height indices between bare footprints and foot impressions on athletic shoe insoles should be aware of the lack of statistical correlation. However, additional research should be done to confirm these findings and to further consider the utility of arch indices in forensic applications. 


\section{References:}

1. J. A. DiMaggio, W. Vernon. Forensic Podiatry: Principles and Methods. 2nd ed., CRC Press, Boca Raton, FL, 2017.

2. C. J. Smerecki, C. O. Lovejoy, Identification via pedal morphology. Int. Crim. Police Rev. 40 (1985) 186-190.

3. S.L. Barker, J.L. Scheuer, Predictive value of human footprints in a forensic context. Med Sci Law 38 (1998) 341-346.

4. K. Krishan, T. Kanchan, J.A. DiMaggio, Emergence of forensic podiatry-a novel sub-discipline of forensic sciences. Forensic Sci. Int. 55 (2015) 16-27.

5. R.B. Kennedy, I.S. Pressman, S. Chen, P.H. Petersen, A.E. Pressman, Statistical analysis of barefoot impressions. J. Forensic Sci. 48(1) (2003) 1-9.

6. R. Mukhra R, K. Krishan, T. Kanchan, Bare footprint metric analysis methods for comparison and identification in forensic examinations: a review of literature. J Forensic Leg. Med. 58 (2018) 101-112.

7. K. Krishan, Individualizing characteristics of footprints in Gujjars of North India forensic aspects, Forensic Sci. Int. 169 (2007) 137-144.

8. R.B. Kennedy, Ongoing research into barefoot impression evidence, in: J. Rich, D.E. Dean, R.H. Powers (Eds.), Forensic Medicine of the Lower Extremity, Humana Press, Totawa 2005, pp. 401-413.

9. R.B. Kennedy, Preliminary study on the uniqueness of barefoot impressions. Can. Soc. Forensic Sci. J. 29 (4) (1996) 233-238. 
10. G.E. Laskowski, K.L. Vernon, Barefoot impressions—a preliminary study of identification characteristics and population frequency of their morphological features. J. Forensic Sci. 33, (2) (1988) 378-388.

11. R.B. Kennedy, C. Sanping, I.S. Pressman, B. Yamashita, A.E. Pressman, A large-scale statistical analysis of barefoot impressions, J. Forensic Sci. 50 (5) (2005) 1071-1080.

12. L. Hammer, N.N. Daéid, R. B. Kennedy, A. B. Yamashita, Preliminary study of the comparison of inked barefoot impressions with impressions from shoe insoles using a controlled population. J. Forensic Identif. 62 (6) (2012) 603-21.

13. M.S. Nirenberg, E. Ansert, K. Krishan, T. Kanchan, Two-dimensional metric comparisons between dynamic bare footprints and insole foot impressionsforensic implications. Sci. Justice Dec 4 (2019).

14.S. Leishman, If the Shoe Fits! RCMP Gazette 53 (1991) 4-7.

15. R.M. Queen, N.A. Mall, M. Hardaker and J.A. Nunley, Describing the medial longitudinal arch using footprint indices and a clinical grading system. Foot ankle int. 28.4 (2007) 456-62.

16. C.H. Chang, Y.C. Chen, W.T. Yang, P.C. Ho, A.W. Hwang, C.H. Chen, J.H. Chang, L.W. Chang, Flatfoot diagnosis by a unique bimodal distribution of footprint index in children. PLoS One 9.12 (2014) e115808.

17. D.L. Riddiford-Harland, J. R. Steele, and L. H. Storlien, Does obesity influence foot structure in prepubescent children? Int. J. Obesity 24.5 (2000) 541. 
18. H. Dadgar, M. Sahebozamani, Evaluation of sole arech (sic) index and noncontact lower extremity injury rates in male karateka (sic). J. Research Rehabil. Sci. 7(1) (2011) 0.

19. I.C.N. Sacco, A.N Onodera, K. Bosch, D. Rosenbaum, Comparisons of foot anthropometry and plantar arch indices between German and Brazilian children. BMC Pediatr 15.1 (2015): 4.

20.S.C. Wearing, A.P. Hills, N.M. Byrne, E.M. Hennig, M. McDonald, The arch index: a measure of flat or fat feet? Foot Ankle Int. 25 (8) (2004) 575-581.

21. H.B. Menz, M. R. Fotoohabadi, E. Wee, M. J. Spink, Visual categorisation of the arch index: a simplified measure of foot posture in older people. J. Foot Ankle Res. 5(1) (2012) 10.

22. S. Pita-Fernández, C. González-Martín, T. Seoane-Pillado, B. López-Calviño, S. Pértega-Díaz, V. Gil-Guillén, Validity of footprint analysis to determine flatfoot using clinical diagnosis as the gold standard in a random sample aged 40 years and older. J. Epi. (2015): JE20140082.

23. S. Pita-Fernández, C. González-Martín, T. Seoane-Pillado, B. López-Calviño, S. Pértega-Díaz, V. Gil-Guillén, Flat foot in a random population and its impact on quality of life and functionality. J. Clin. Diag. Res. JCDR 11(4), (2017) LC22.

24.J.S. Wrobel, D. G. Armstrong, Reliability and validity of current physical examination techniques of the foot and ankle. J. Am. Podiat. Med. Assn. 98 (3) (2008) 197-206 
25. J.J. Echarri, F. Francisco, The development in footprint morphology in 1851 Congolese children from urban and rural areas, and the relationship between this and wearing shoes. J. Ped. Orth. B 12.2 (2003) 141-146.

26. I. Mathieson, D. Upton, A. Birchenough, Comparison of footprint parameters calculated from static and dynamic footprints. The Foot 9 (3) (1999) 145-149.

27.S. C. Kippen, "A preliminary assessment of recording the physical dimensions of an inked footprint." J. Brit. Pod. Med. 48 (1993) 74-80.

28. B.Y.S. Tsung, M. Zhang, Y. B. Fan, D. A. Boone, Quantitative comparison of plantar foot shapes under different weight-bearing conditions. J. Rehab. Res. Dev. 40 (6) (2003) 517-526.

29. Commonwealth of Virginia v. Stanfield, 2015 GC14000548-00. News item Available at: https://pilotonline.com/news/local/crime/article_a8e9dfd1- b1275621-908e-03dbb594f530.html (accessed on 9th June 2019).

30. A. Janos, What's a 'Shoe Autopsy'? A Forensic Podiatrist Explains the Value of Foot Evidence in Solving Crimes. Real Crime. A\&E Network. https://www.aetv.com/real-crime/forensic-podiatry-interview-foot-evidencefootprints-crime-solving (accessed 3 January 2020).

31. Forensic Files: A Cinderella Story. Medstar Television, Season 12, Episode 11, aired 24 December 2007.

32. M.S. Nirenberg, E. Ansert, K. Krishan, T. Kanchan, Two-dimensional metric comparison between dynamic bare and sock-clad footprints for its forensic implications-A pilot study. Sci. Justice 59, no. 1 (2019) 46-51. 
33. M.S. Nirenberg, E. Ansert, K. Krishan, T. Kanchan, Two-dimensional linear analysis of dynamic bare footprints: A comparison of measurement techniques. Sci. Justice Apr. 1 (2019).

34. M. Morlock, T. Mittlmeiser, First step method vs. full gait method - results of a comparison. Eur .J. Phys. Med. Rehab. (Suppl 1) 2 (1992) 33-34.

35. S.C. Wearing, S.R. Urry, J.E. Smeathers, D. Battistutta, A comparison of gait initiation and termination methods for obtaining plantar foot pressures. Gait Posture, 10 (1999) 255-263.

36. N Howsam, A. Bridgen, A comparative study of standing fleshed foot and walking and jumping bare footprint measurements. Sci. Justice 58.5 (2018) 346354.

37. S. Reel, S. Rouse, W. Vernon, P. Doherty, Reliability of a two-dimensional footprint measurement approach. Sci. Justice, 50 (2010) 113-118.

38.P.J. McNair, R. N. Marshall, Kinematic and kinetic parameters associated with running in different shoes. Brit. J. Sports Med. 28.4 (1994) 256-260.

39. E. M. Hennig, Eighteen years of running shoe testing in Germany-a series of biomechanical studies. Footwear Sci. 3.2 (2011) 71-81.

40. M.R. Shorten, Running shoe design: protection and performance, in D.T. Pedoe (Ed.), Marathon medicine, Royal Society of Medicine, London, 2000, pp. 159169. 
Forensic Implications of Foot Arch Index Comparison between Dynamic Bare

Footprints and Shoe Insole Foot Impressions Figures, Tables, and Captions

Figure 1: Measurements made on the bare footprints.

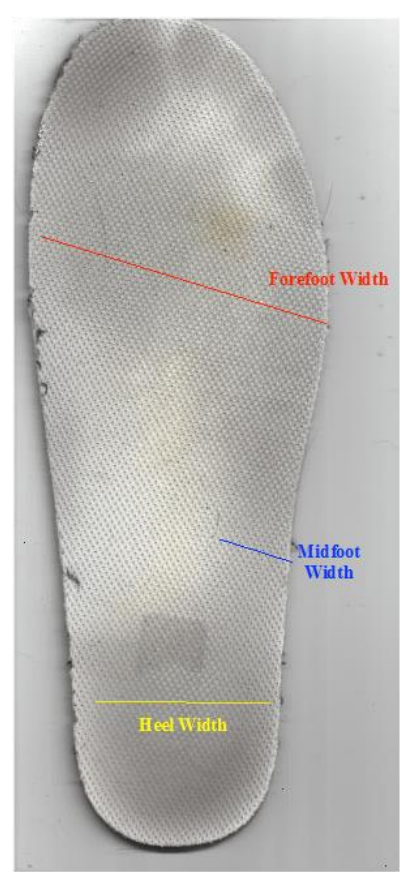

Figure 2: Measurements made on the shoe insole impressions. 


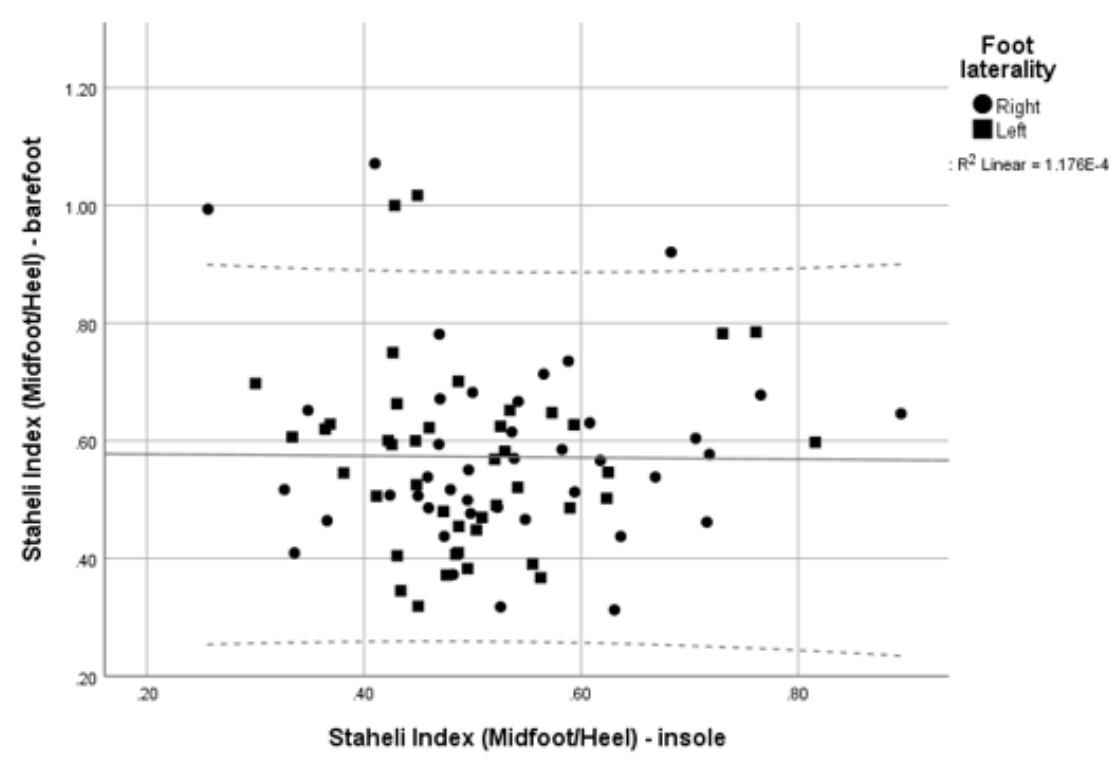

Figure 3: Scatter plot of Staheli arch height index from insole foot impression against Staheli index from bare footprint. Solid line shows regression line. Dotted lines show 95\% confidence interval for individual predictions.

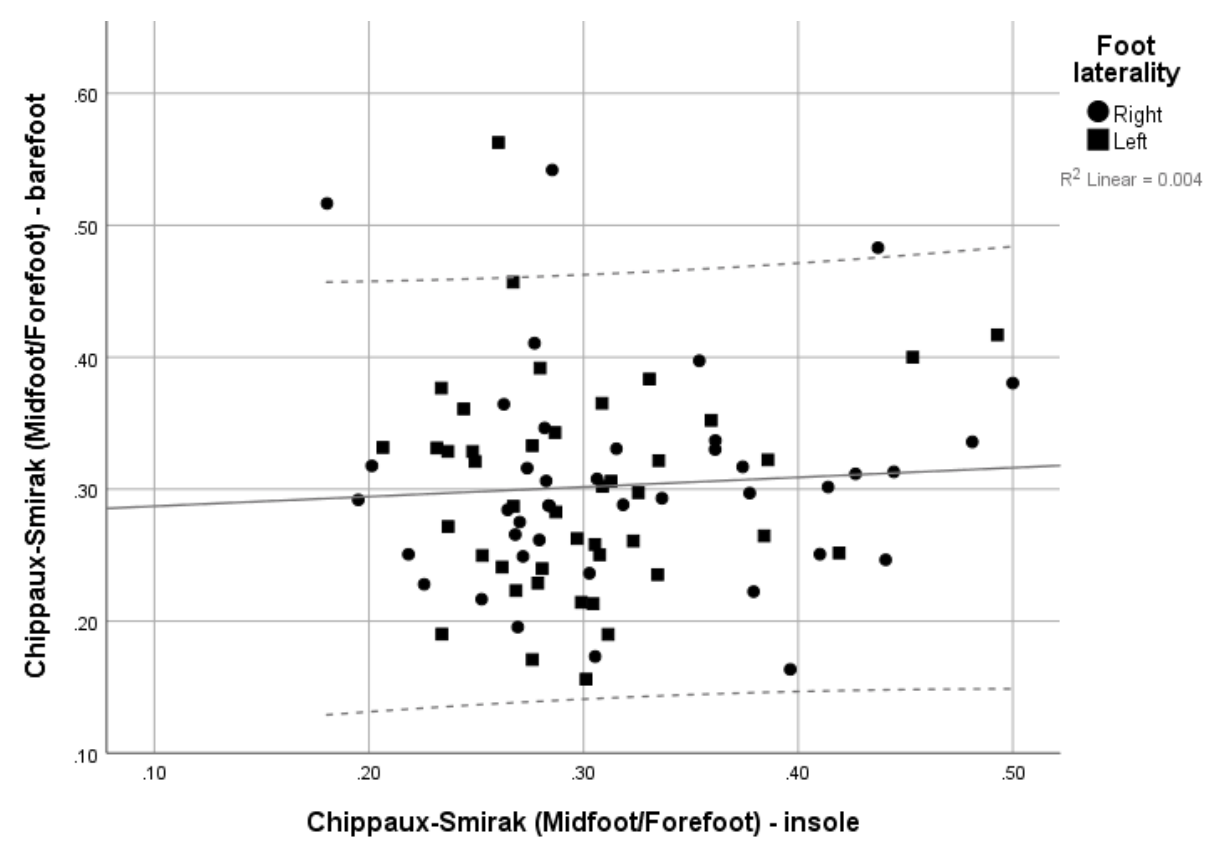

Figure 4: Scatter plot of Chippaux-Smirak arch height index from insole foot impression against Chippaux-Smirak index from bare footprint. Solid line shows regression line. Dotted lines show 95\% confidence interval for individual predictions. 


\section{Credit Author Statement}

Michael Nirenberg, DPM (corresponding author): conceptualization, methodology, writing review and editing, project administration

Elizabeth Ansert, DPM, MA: methodology, investigation, writing original draft, writing review and editing

Jackie Campbell, PhD MSc BSc CStat CPhys FRCOT FRSB: data curation, writing review and editing

Michael Curran, Ph.D, M.Phil, MBA, BSc(Hons): methodology, writing review and editing 